\title{
Utilizing fourth-generation endocytoscopy and the 'enlarged nuclear sign' for in vivo diagnosis of early gastric cancer
}

\section{(ㄷ)(1) $\Theta$}

\author{
Authors \\ Mary Raina Angeli Abad, Haruhiro Inoue, Haruo Ikeda, Anastassios Manolakis, Enrique Rodriguez de Santiago, \\ Ashish Sharma, Yusuke Fujiyoshi, Hisashi Fukuda, Kazuya Sumi, Manabu Onimaru, Yuto Shimamura
}

Institution

Digestive Diseases Center, Showa University Koto Toyosu Hospital, Tokyo, Japan

submitted 28.1.2019

accepted after revision 2.5.2019

Bibliography

DOI https://doi.org/10.1055/a-0957-2866 |

Endoscopy International Open 2019; 07: E1002-E1007

(c) Georg Thieme Verlag KG Stuttgart · New York

eISSN 2196-9736

Corresponding author

Mary Raina Angeli Abad, MD, Digestive Diseases Center, Showa University Koto Toyosu Hospital, 5-1-38 Toyosu, Koto-ku, Tokyo 135-8577, Japan

Fax: +81-3-6204-6396

raina.abadmd@gmail.com

\section{ABSTRACT}

Background and study aims Fourth-generation endocytoscopy is an ultra-high magnification endoscopic technique designed to provide excellent quality in vivo histologic assessment of gastrointestinal lesions. This study aims to evaluate the diagnostic accuracy of endocytoscopy in early gastric cancer diagnosis.

Patients and methods A single-center, retrospective analysis of prospectively collected data from all gastric endocytoscopic examinations was conducted. Two expert endo- scopists, blinded to white-light and narrow-band imaging findings as well as histopathologic diagnosis, independently reviewed and diagnosed all endocytoscopic images. A newly recognized "enlarged nuclear sign" was detected, and its implication in early gastric cancer diagnosis was evaluated. The diagnostic performance of fourth-generation endocytoscopy was assessed while using the gold standard histopathology as a reference.

Results Forty-three patients (mean age \pm SD, 72.6 \pm 12.1 years; 31 males) were enrolled. Based on histopathology, 23 had well-differentiated adenocarcinomas, four adenomas, and 16 non-neoplastic lesions. The sensitivity, specificity, and accuracy of fourth-generation endocytoscopy for gastric cancer diagnosis were $87.0 \%$ (95\% Cl: 67.9-95.5), $80.0 \%$ (95\% Cl: $58.4-91.9)$, and $83.7 \%$ (95\% Cl: $70.0-$ 91.9) by endoscopist $\mathrm{A}$; and $91.3 \%$ (95\% Cl: $73.2-97.6)$, $75.0 \%$ (95\% Cl: $53.1-88.8)$, and $83.7 \%(95 \% \mathrm{Cl}: 70.0-$ 91.9) by endoscopist B. The inter-observer agreement, Kappa statistic $=0.71(95 \% \mathrm{Cl}: 0.50-0.93)$, was good. The sensitivity, specificity, and accuracy of the enlarged nuclear sign for early gastric cancer diagnosis were $87.0 \%(95 \% \mathrm{Cl}$ : 67.9 -95.5), $95.0 \%$ (95\% Cl: $76.4-99.1$ ), and $90.7 \%$ (95\% $\mathrm{Cl}$ : 78.4-96.3) by endoscopist $\mathrm{A}$; and $82.6 \%(95 \% \mathrm{Cl}$ : $62.9-93.0), 85.0 \%$ (95\% Cl: $64.0-94.8)$, and $83.7 \%$ (95\% $\mathrm{Cl}: 70.0-91.9)$ by endoscopist B. The inter-observer agreement, Kappa statistic $=0.68(95 \% \mathrm{Cl}: 0.51-0.89)$ was good . Conclusion: Fourth-generation endocytoscopy appears to aid in the diagnosis of early gastric cancer, particularly welldifferentiated adenocarcinomas, due to its good diagnostic accuracy and identification of the "enlarged nuclear sign," and deserves further evaluation in future studies.

\section{Introduction}

Gastric cancer is the fifth most commonly diagnosed cancer and the third leading cause of cancer-related mortality [1]. Despite a worldwide decrease in incidence and mortality, gastric cancer remains quite prevalent in parts of Asia, Eastern Europe, and South America [1]. Therefore, early detection and diagnosis of gastric cancer remains a key element for improved prognosis and survival outcomes.
Although the gold standard for diagnosis of gastric cancer is histopathological examination [2], recent endoscopic technologies have been developed to optimize detection and diagnosis of early-stage gastric cancer. Among these, magnifying endoscopy with narrow-band imaging (NBI) and chromoendoscopy are more commonly applied whereas others such as confocal endomicroscopy and endocytoscopy, although promising, are rarely used. 
Endocytoscopy (EC) is an ultra-high magnification endoscopic technique that allows microscopic visualization of the mucosal surface of gastrointestinal cells by using intraprocedural stains [3]. First-generation EC was developed in 2003 [4, 5]. Since then, several modifications and refinements allowed the development of fourth-generation endocytoscopes that exhibit a magnification power of $\times 500$, thus enabling in vivo tissue observation, and visualization of individual cell nuclei $[4,5]$.

To date, EC has been used for assessment of pharyngeal, esophageal, duodenal, and colonic lesions. However, there are a limited number of studies examining gastric lesions because the quality of EC images in the stomach is far from excellent due to poor staining, resulting from presence of gastric secretions [6]. Bearing that in mind, the idea of performing a diagnostic accuracy study utilizing fourth-generation EC while at the same time addressing these issues seemed quite appealing. This study primarily aimed to evaluate the diagnostic performance and accuracy of EC in the diagnosis of early gastric cancer.

\section{Patients and methods}

\section{Study population}

This is a retrospective analysis of data collected prospectively at a single tertiary referral center, Showa University Koto Toyosu Hospital, between December 2015 and October 2018. All individuals who underwent gastric EC examination and magnifying endoscopy work-up before or after endoscopic submucosal dissection (ESD) were included.

\section{Endocytoscopy technical considerations}

EC examinations were carried out using a fourth-generation endocytoscope with a single lens reflection, consecutive increase in magnification to $\times 500$, observation range of $570 \times 500 \mu \mathrm{m}$, and an outer diameter of $9.7 \mathrm{~mm}$ (GIF-H290EC; Olympus Medical Systems Corp., Tokyo, Japan). A black silicone cap (Distal Hood MAJ-1989; Olympus Medical Systems Corp., Tokyo, Japan) was placed at the tip of the endocytoscope, secured by a white tape in an oblique manner in order for the lens to come in contact with the gastric mucosa.

\section{Endocytoscopic assessment}

All gastric EC examinations were performed under intravenous sedation using propofol $(200 \mathrm{mg} / 20 \mathrm{~mL})$ by board-certified fellows of the Japan Gastroenterological Endoscopy Society. All
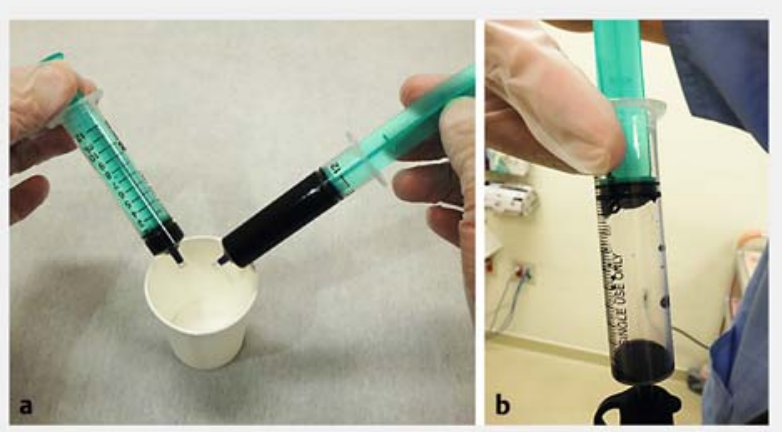

- Fig. 1 CM double staining. a An 11-cc staining solution is prepared consisting of $10 \mathrm{cc}$ of crystal violet and $1 \mathrm{cc}$ of methylene blue. $\mathbf{b}$ In a separate 10 -cc syringe, $1 \mathrm{cc}$ of the solution is aspirated with $9 \mathrm{cc}$ of air and is used for spraying.

subjects received $40 \mathrm{~mL}$ of water-based solution also containing the mucolytic agent pronase (20000 U; Kaken Pharmaceutical, Tokyo, Japan) and the defoaming substance dimethicone (Balgin 2\% Antifoaming Oral Solution; Kaigen Pharmaceutical, Tokyo, Japan) per os prior to endoscopy. The suitable lesions were initially examined with white-light endoscopy (WLE) and $\mathrm{NBI}$, followed by EC using $0.05 \%$ crystal violet and $0.1 \%$ methylene blue (CM) double staining. The staining solution is a mixture of 10 cc crystal violet and 1 cc methylene blue. In two to three separate 10 -cc syringes, $1 \mathrm{cc}$ each is aspirated with $9 \mathrm{cc}$ of air ( $\triangleright$ Fig. 1 ) which is then used for spraying multiple times until a satisfactory staining is achieved. The average total procedure time was approximately 30 to 40 minutes, with endocytoscopy taking around 15 to 20 minutes. All images were retrieved from the database and an all-EC image catalog was created. EC images were assessed separately by two expert endoscopists, with expertise in third-generation EC (>50 x examinations), blinded to patient's clinical information, WLE and NBI findings, and histopathological results. EC diagnosis was made by assessing cellular and glandular morphology, lumen changes, loss of polarity of nuclei, and nuclear structural changes, and was based on an adaptation of EC classification, originally created for colorectal lesions [7], for gastric mucosa ( $\triangleright$ Table 1). In brief, EC1 lesions are considered as non-neoplastic, EC2 as adenomas, and EC3 as carcinomas ( $>$ Fig. 2). Hyperchromatic nuclei occupying the vast majority of the cell that are disar-

- Table 1 EC classification of gastric mucosa.

\begin{tabular}{|c|c|c|c|}
\hline Classification & \multicolumn{2}{|l|}{ Structure } & Nuclei \\
\hline $\begin{array}{l}\text { EC1 } \\
\text { (Non-neoplastic) }\end{array}$ & \multicolumn{2}{|c|}{$\begin{array}{l}\text { Smooth surfaces, soft edges, regular pit pattern, wide } \\
\text { lumen }\end{array}$} & $\begin{array}{l}\text { Regular pattern and arrangement of small uniformly sized, } \\
\text { rounded nuclei with poor staining }\end{array}$ \\
\hline $\begin{array}{l}\text { EC2 } \\
\text { (Adenoma) }\end{array}$ & \multicolumn{2}{|c|}{ Dense slit-like lumen (lumen narrowing) } & $\begin{array}{l}\text { Regular pattern of small, uniformly sized, rounded nuclei } \\
\text { with poor staining }\end{array}$ \\
\hline $\begin{array}{l}\text { EC3 } \\
\text { (Cancer) }\end{array}$ & $\begin{array}{l}\text { Well-differentiated } \\
\text { adenocarcinoma }\end{array}$ & $\begin{array}{l}\text { Irregular, rough, distorted } \\
\text { gland formation, absence } \\
\text { or fusion of lumen }\end{array}$ & $\begin{array}{l}\text { Hyperchromatic, disarranged, swollen nuclei with hetero- } \\
\text { genous shape (large and elongated, large with rough edges) } \\
=\text { "enlarged nuclear sign" }\end{array}$ \\
\hline
\end{tabular}



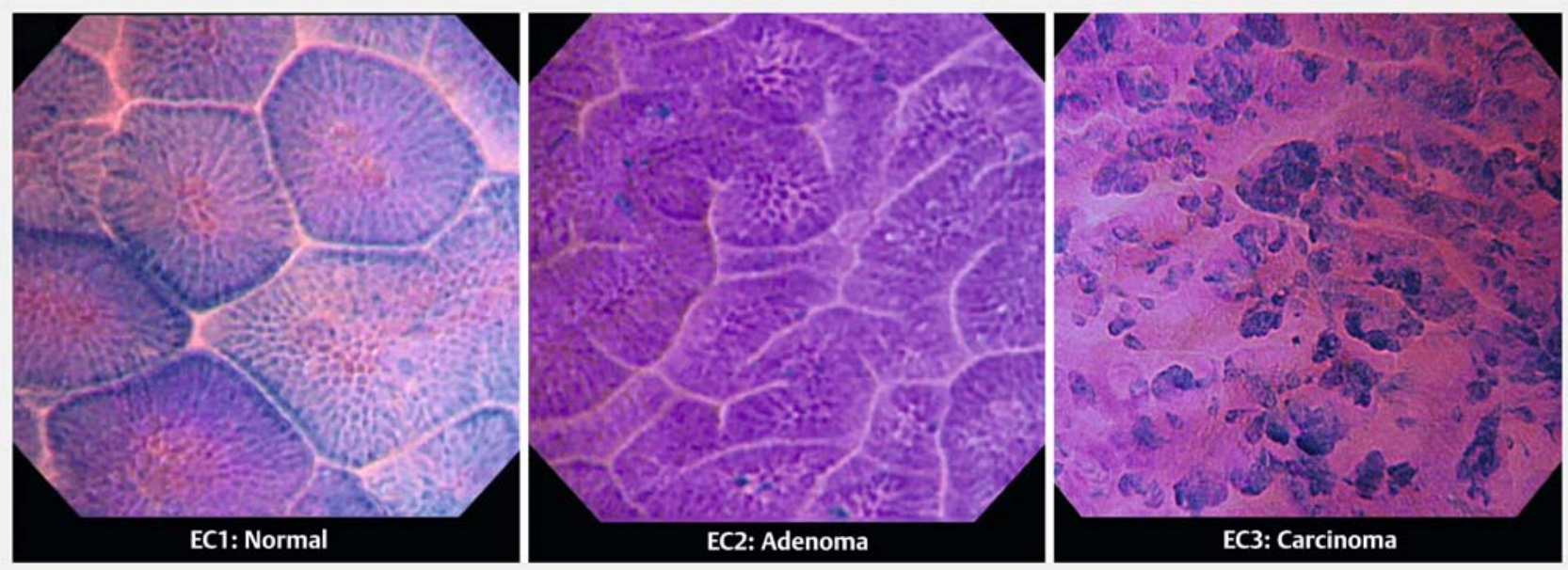

Fig. 2 EC classification of gastric mucosa. Representative pictures differentiating EC1 (normal), EC2 (adenoma), and EC3 (carcinomas).

ranged and with heterogenous shape (large and elongated, large with rough edges) were detected in the process and termed as the "enlarged nuclear sign" (ENS). The efficiency of ENS in distinguishing between malignant and non-malignant lesions was also evaluated by the two expert endoscopists. Biopsy specimens were available for all cases, however, for gastric cancer, histopathologic diagnosis was made on ESD specimens. Accuracy of expert EC-based diagnosis was assessed while using the gold standard histopathology as a reference.

\section{Statistical analysis}

The Shapiro-Wilk test and distributional plots were used to assess normality. Continuous variables are reported as mean \pm standard deviation (SD) when normality was met. Categorical variables are described as absolute and relative frequencies. Sensitivity, specificity, and accuracy for diagnosis of early gastric cancer were calculated for each endoscopist. The Kappa statistic was used to estimate the inter-observer agreement. Kappa values of $\leq 0.40,0.41-0.75$, and $\geq 0.75$ were considered poor, good, and excellent, respectively. The Wilson method was used to calculate $95 \%$ confidence intervals. All analyses were performed using STATA software version 14.1 (StataCorp. Texas, United States).

\section{Ethical considerations}

The study protocol adhered to the principles of the Declaration of Helsinki and was approved by the Ethics Committee of Showa University Koto Toyosu Hospital (IRB Registration No: 17T7040). Written informed consent was obtained from all participants.

\section{Results}

A total of 43 patients (mean age \pm SD, $72.6 \pm 12.1$ years, 31 ( $72 \%$ ) males) were enrolled. Population characteristics are presented in $>$ Table 2. Based on histopathology, 23 were diagnosed with well-differentiated adenocarcinomas, four with adenomas, and 16 with non-neoplastic lesions. The non-neoplastic lesions com-
- Table 2 Patient clinicopathological characteristics $(n=43)$.

\begin{tabular}{|c|c|c|}
\hline Variable & $\begin{array}{l}\text { Non-malignancy } \\
(n=20)\end{array}$ & $\begin{array}{l}\text { Malignancy } \\
(\mathrm{n}=23)\end{array}$ \\
\hline Age (mean age $\pm S D$ ) & $69 \pm 14.07$ & $75.25 \pm 9.44$ \\
\hline \multicolumn{3}{|l|}{ Sex } \\
\hline - Male & 14 & 17 \\
\hline - Female & 6 & 6 \\
\hline \multicolumn{3}{|l|}{ Macroscopic Type } \\
\hline . $0-1$ & 1 & 2 \\
\hline - 0-IIa & 2 & 8 \\
\hline . $0-I I b$ & 2 & 1 \\
\hline - 0 -IIc & 2 & 12 \\
\hline . $0-111$ & 0 & 0 \\
\hline \multicolumn{3}{|l|}{ Location } \\
\hline - Corpus & 6 & 13 \\
\hline - Antrum & 2 & 8 \\
\hline - Cardia & 1 & 2 \\
\hline \multicolumn{3}{|l|}{ Histopathology } \\
\hline $\begin{array}{l}\text { - Non-neoplastic gastric } \\
\text { mucosa }\end{array}$ & $16(37.2 \%)$ & $\mathrm{N} / \mathrm{A}$ \\
\hline - Adenoma & $4(9.3 \%)$ & $\mathrm{N} / \mathrm{A}$ \\
\hline - Tubular adenocarcinoma & $N / A$ & $23(53.5 \%)$ \\
\hline
\end{tabular}

SD, standard deviation; N/A, not applicable.

prised two hyperplastic polyps, two chronic gastritis, one hyperplastic mucosa, and 11 normal gastric mucosa.

Based upon expert endoscopists' ratings, high-quality images could be obtained in $81.4 \%$ of cases. Comparison of EC diag- 
- Table 3 Comparison of expert EC-based diagnosis and histopathologic diagnosis.

\begin{tabular}{|c|c|c|c|c|c|c|}
\hline \multirow[t]{3}{*}{ Histopathologic diagnosis } & \multicolumn{6}{|c|}{ Endocytoscopic diagnosis } \\
\hline & \multicolumn{3}{|c|}{$\begin{array}{l}\text { Endoscopist A } \\
(n=43)\end{array}$} & \multicolumn{3}{|c|}{$\begin{array}{l}\text { Endoscopist B } \\
(n=43)\end{array}$} \\
\hline & EC1 & EC2 & EC3 & EC1 & EC2 & EC3 \\
\hline Non-neoplastic & 13 & 2 & 1 & 12 & 2 & 2 \\
\hline Adenoma & 0 & 1 & 3 & 0 & 1 & 3 \\
\hline Cancer & 0 & 3 & 20 & 0 & 2 & 21 \\
\hline
\end{tabular}

- Table4 Diagnostic yields of endocytoscopy for early gastric cancer.

\begin{tabular}{|l|l|l|l|l|}
\hline \multirow{2}{*}{ Test } & \multicolumn{2}{|c|}{ Endoscopist A } & \multicolumn{2}{c|}{ Endoscopist B } \\
\cline { 2 - 5 } & Results & $\mathbf{9 5 \%} \mathbf{C l}$ & Results & $\mathbf{9 5 \% ~ C l}$ \\
\hline Sensitivity & $87.0 \%$ & $67.9-95.5$ & $91.3 \%$ & $73.2-97.6$ \\
\hline Specificity & $80.0 \%$ & $58.4-91.9$ & $75.0 \%$ & $53.1-88.8$ \\
\hline Accuracy & $83.7 \%$ & $70.0-91.9$ & $83.7 \%$ & $70.0-91.9$ \\
\hline
\end{tabular}

$\mathrm{Cl}$, confidence interval.

- Table 5 Diagnostic yields of enlarged nuclear sign for early gastric cancer.

\begin{tabular}{|l|l|l|l|l|}
\hline \multirow{2}{*}{ Test } & \multicolumn{2}{|c|}{ Endoscopist A } & \multicolumn{2}{c|}{ Endoscopist B } \\
\cline { 2 - 5 } & Results & $\mathbf{9 5 \%} \mathbf{C l}$ & Results & $\mathbf{9 5 \%} \mathrm{Cl}$ \\
\hline Sensitivity & $87.0 \%$ & $67.9-95.5$ & $82.6 \%$ & $62.9-93.0$ \\
\hline Specificity & $95.0 \%$ & $76.4-99.1$ & $85.0 \%$ & $64.0-94.8$ \\
\hline Accuracy & $90.7 \%$ & $78.4-96.3$ & $83.7 \%$ & $70.0-91.9$ \\
\hline
\end{tabular}

$\mathrm{Cl}$, confidence interval.

nosis made by the two experts is presented in $>$ Table 3 . Based on these data, the sensitivity, specificity, and accuracy of EC for diagnosis of early gastric cancer were calculated, as shown in - Table 4. EC findings of 11 normal gastric mucosa showed regular glands, smooth surfaces, and soft edges, well-preserved lumen, and small uniformly sized rounded nuclei with poor staining ( Fig. 3 ). No infiltrating cells, necrotic tissue, or debris were identified. Chronic gastritis revealed more stained crypts and presence of infiltrating cells and debris, with regular glands and well-preserved lumen. Hyperplastic polyps demonstrated wider, oval lumen with small uniformly sized rounded nuclei.

Average sensitivity, specificity, and accuracy of EC for both endoscopists were $89.0 \%, 77.5 \%$, and $83.7 \%$, respectively. Interobserver agreement, Kappa statistic $=0.71(95 \% \mathrm{Cl}: 0.50-$ $0.93)$, was good.

ENS was observed in malignant lesions and its sensitivity, specificity, and accuracy for diagnosis of early gastric cancer were calculated and presented in $>$ Table 5 . Average sensitivity,
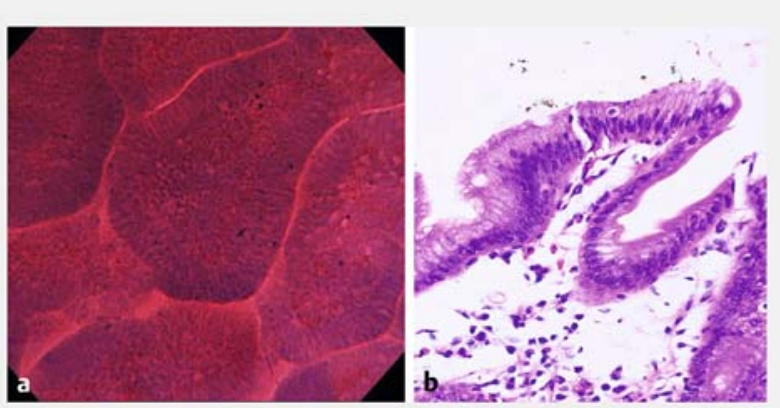

- Fig. 3 Normal gastric mucosa. a Endocytoscopic findings of normal gastric mucosa showing regular glands, smooth surfaces and soft edges, well-preserved lumen, and small uniformly sized rounded nuclei with poor staining. b Histologic appearance of normal gastric mucosa

specificity, and accuracy of ENS for both endoscopists were $84.8 \%, 90.0 \%$, and $87.2 \%$, respectively. Interobserver agreement, Kappa statistic $=0.68(95 \% \mathrm{Cl}: 0.51-0.89)$, was good .

\section{Discussion}

Diagnostic performance and accuracy of fourth-generation EC to discern early gastric cancer among endoscopically identified lesions was evaluated in this study. When compared to the gold-standard histopathology, fourth-generation EC showed satisfactory accuracy in diagnosing early gastric cancer. Identification of ENS seems to further aid the differentiation of gastric cancer from non-malignancy.

Although the concept of EC as a tool for gastric cancer diagnosis is not entirely novel, the number of available studies on the subject is rather limited. The scarcity of relevant data can be attributed to the challenges related to obtaining good-quality staining as well as limited availability of the necessary dedicated endocytoscopes, which are only available in a small number of centers worldwide. As the gastric mucosa is exhibiting enhanced mucus secretory function, the abundance of mucus poses a challenge for high-quality staining. In the current study, this issue was addressed by applying the mucolytic pronase prior to the procedure as well as careful water-jet-assisted mucosal rinsing using water plus dimethicone solution before $\mathrm{CM}$ 
double staining multiple times through the scope channel. The combination of the cutting-edge fourth-generation endocytoscope, which provides higher magnification, with CM to produce a staining pattern that resembles the traditional hematoxylin-eosin (H\&E) stain [8-11], allowed acquisition of high-quality images in over $80 \%$ of cases in this study.

By obtaining images of higher quality, identification of tissue and cellular characteristics was optimized, thus leading to more accurate detection of morphological changes associated with cancer (distortion in gastric tissue architecture, cellular atypia, etc.) ( $\mathbf{F i g . 4 )}$. Based on presence or absence of these EC-identified changes, three distinct diagnostic entities could be identified corresponding to traditional classification of lesions as being non-neoplastic, neoplastic yet benign, or malignant. Through adaptation of a previous EC classification that was originally used in colorectal lesions [7], a novel EC classification, this time for the gastric mucosa, was created.

The combination of favorable characteristics (images of high quality and magnification) with EC classification of gastric mucosa led to good diagnostic accuracy and interobserver agreement for distinguishing between gastric cancer and nonmalignant cases in our study. Interestingly, the results were comparable to those acquired through standard histopathology with the exception of adenomas, for which a trend towards over-grading was recorded. Safe conclusions regarding this potential over-grading of adenomas cannot be drawn, mainly due to the very limited number of adenoma cases in this study.

Another major finding in our study was detection of disarranged, heterogenous-shaped, hyperchromatic nuclei large enough to give the impression of "taking over" the entire cell surface ( $\triangleright$ Fig.5). Although the nucleus:cytoplasm ( $\mathrm{N}: \mathrm{C}$ ) ratio has been traditionally used during histopathologic as well as endocytoscopic evaluation $[2,6,12,13]$, the striking characteristic of nuclear "taking over" observed in the EC images of gastric adenocarcinoma ( $>$ Fig. 6) in this study encouraged its use as a distinct feature, and the term "enlarged nuclear sign" was coined to describe it. Using ENS provided good diagnostic accuracy and interobserver agreement for differentiation of gastric cancer from non-malignancy, thus supporting its further use for this purpose.

At this point, however, certain study limitations must be acknowledged. To avoid bias due to preceding WLE and NBI assessment, only still EC images were retrieved from the database and assessed by the two expert endoscopists. Moreover, the sample size is relatively small, suggesting that future studies on the same topic may necessitate a larger number of patients to substantiate the results of this study. Non-neoplastic lesions that mimic well-differentiated adenocarcinomas in histopathology were not encountered in this study, therefore, EC assessment and differentiation between these lesions and adenocarcinomas could not be determined. Finally, all gastric malignancy cases in this study pertained to well-differentiated adenocarcinomas, therefore, no conclusions can be drawn about EC assessment of poorly-differentiated adenocarcinomas.
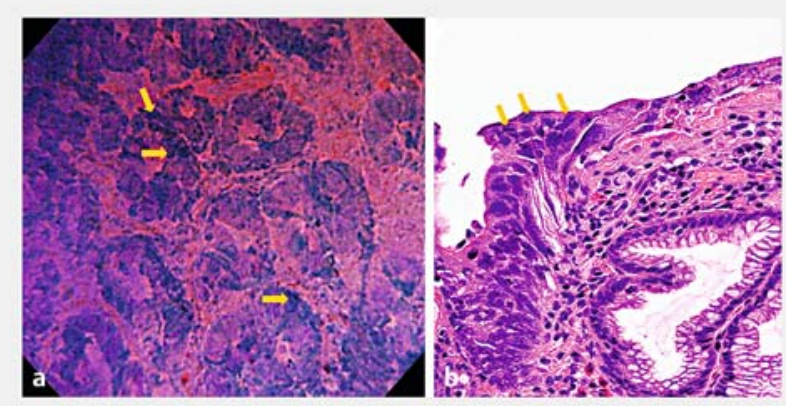

- Fig. 4 Well-differentiated adenocarcinoma. a Endocytoscopic findings of well-differentiated adenocarcinoma showing irregular and distorted glands and the strongly stained "enlarged nuclear sign" (yellow arrows). b Histologic appearance of well-differentiated adenocarcinoma.
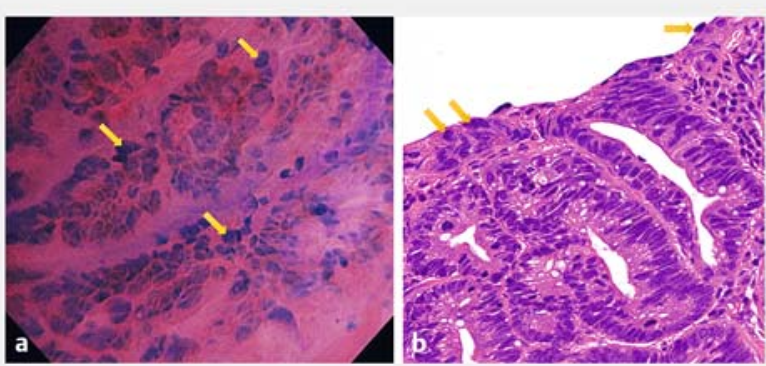

- Fig. 5 "Enlarged nuclear sign". Representative pictures of the "enlarged nuclear sign" (yellow arrows) by endocytoscopy a and by histopathology $\mathbf{b}$ depicting the striking characteristic of nuclear "taking over" the cell surface.
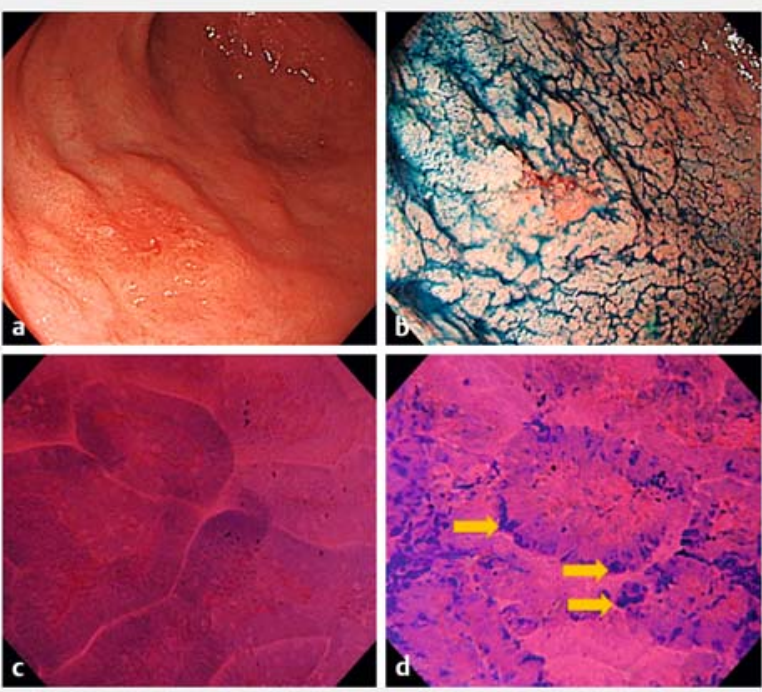

- Fig. 6 Early gastric cancer case diagnosed using fourth-generation endocytoscopy. a, b A lesion in the antrum observed by whitelight endoscopy. c Endocytoscopic findings revealed non-neoplastic glands of the surrounding mucosa, $\mathbf{d}$ while the highly dysplastic area showed distortion of glands and presence of the "enlarged nuclear sign" (yellow arrows). 


\section{Conclusions}

In all, due to its good diagnostic accuracy and identification of the newly-recognized "enlarged nuclear sign" in this study, fourth-generation EC seems to aid in diagnosis of early gastric cancer, particularly well-differentiated adenocarcinomas, and deserves further evaluation in future studies.

\section{Competing interests}

Dr. Inoue is an advisor of Olympus Corporation and Top Corporation. He has also received educational grants from Olympus Corp., and Takeda Pharmaceutical Co.Dr. Manolakis is a Hellenic Society of Gastroenterology grant holder. Dr. Rodriguez de Santiago is a Ramón y Cajal Health Research Institute grant holder.

\section{References}

[1] Bray F, Ferlay J, Soerjomataram I et al. Global cancer statistics 2018: GLOBOCAN estimates of incidence and mortality worldwide for 36 cancers in 185 countries. Ca Cancer J Clin 2018; 0: 1- 31

[2] Tsurodome I, Miyahara R, Funasaka K et al. In vivo histological diagnosis for gastric cancer using endocytoscopy. World J Gastroenterol 2017; 23: $6894-6901$

[3] Kaise M, Ohkura Y, lizuka T et al. Endocytoscopy is a promising modality with high diagnostic accuracy for gastric cancer. Endoscopy 2015; 47: 19-25
[4] Kumagai Y, Kawada K, Takubo K et al. Ultra-high magnification endoscopy (endocytoscopy system) for examination of esophageal lesions. Gastroenterol Endosc 2017; 59: 209-218

[5] Kumagai Y, Takubo K, Kawada K et al. A newly developed continuous zoom-focus endocytoscope. Endoscopy 2017; 49: 176-180

[6] Kaise M, Kimura R, Nomura K et al. Accuracy and concordance of endocytoscopic atypia for the diagnosis of gastric cancer. Endoscopy 2014; 46: 827-832

[7] Kudo SE, Wakamura K, Ikehara $\mathrm{N}$ et al. Diagnosis of colorectal lesions with a novel endocytoscopic classification - a pilot study. Endoscopy 2011; 43: 869-875

[8] Inoue H, Yokoyama A, Kudo SE. Ultrahigh magnifying endoscopy: development of CM double staining for endocytoscopy and its safety. Nihon Rinsho 2010; 68: 1247 - 1252

[9] Ichimasa K, Kudo SE, Mori Y et al. Double staining with crystal violet and methylene blue is appropriate for colonic endocytoscopy: an in vivo prospective pilot study. Dig Endosc 2014; 26: $403-408$

[10] Minami H, Inoue H, Yokoyama A et al. Recent advancement of observing living cells in the esophagus using $\mathrm{CM}$ double staining: endocytoscopic atypia classification. Dis Esophagus 2012; 25: 235-241

[11] Inoue H, Kudo SE, Shiokawa A. Novel endoscopic imaging techniques toward in vivo observation of living cancer cells in the gastrointestinal tract. Clin Gastroenterol Hepatol 2005; 3: S61-S63

[12] Inoue H, Kazawa T, Sato $\mathrm{Y}$ et al. In vivo observation of living cancer cells in the esophagus, stomach, and colon using catheter-type contact endoscope, "endo-cytoscopy system". Gastrointest Endosc Clin N Am 2004; 14: 589 - 594

[13] Sato $H$, Inoue $H$, Hayee B et al. In vivo histopathology using endocytoscopy for non-neoplastic changes in the gastric mucosa: a prospective pilot study. Gastrointest Endosc 2015; 81: 875-881 